\title{
Patientenschulung „Fit für Dialyse“ Ängste abbauen, Fragen beantworten, Kontakte aufbauen
}

Wenn der Patient die Diagnose „Niereninsuffizienz, Sie müssen an die Dialyse“ erhält, fällt er oft emotional in ein tiefes Loch. Viele Fragen und Probleme bleiben unbeantwortet und offen. Die Unsicherheit, wie es nun weitergeht, bleibt zurück. Eine gute Möglichkeit für Patienten sich zu informieren und mit anderen Betroffenen zu sprechen, ist das Patientenschulungsprogramm „Fit für Dialyse“, das bundesweit stattfindet. Es bietet Patienten auch die Chance, Ängste abzubauen und Kontakte zum Pflegepersonal aufzubauen.

Das Programm ist sehr informativ und beinhaltet folgende Themen:

- die Niere allgemein

- Erkrankungen der Niere

- Therapie: Hämodialyse, Peritonealdialyse, Nierentransplantation

- Ernährung

- soziales Recht

\section{Wegweiser für den Alltag}

„Fit für Dialyse“ ist ein einfaches, unkompliziertes Schulungsprogramm für neue - und auch bereits erfahrene - Patienten sowie Angehörige und findet bundesweit statt. In der Patientenschulung vermitteln Fachpersonal aus Dialyseeinrichtungen und aus dem operativen Bereich sowie erfahrene Patienten Informationen ohne Fachlatein, um den Kontakt zu Mitbetroffenen und dem Pflegepersonal zu optimieren. Bestehende Ängste sollen abgebaut und

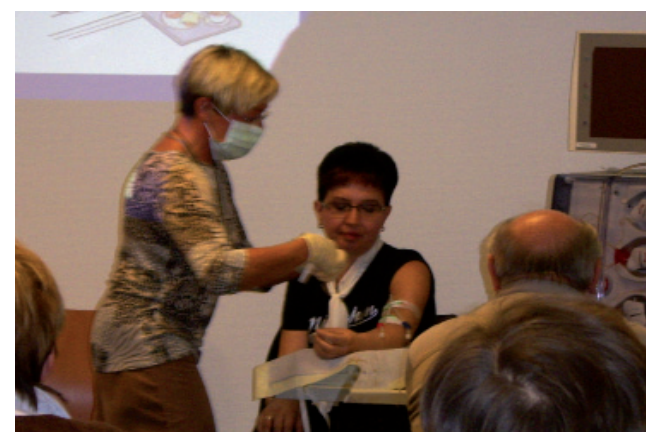

Hintergrundinformationen

Dieses Schulungsprogramm wurde auch als Weiterbildung von Pflegepersonal aus dem Bereich der Dialyse und Transplantation genutzt. In Thüringen wurden bis 2008 bereits 150 Patienten geschult. Die nächste Schulung findet voraussichtlich im September 2009 statt. Mehr Informationen im Internet unter www.fit-fuerdialyse.de

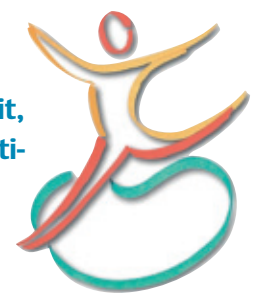

Das Schulungsprogramm findet seit 6 Jahren statt. Wie die bisherigen Erfahrungen zeigen, nehmen die Patienten allerdings nur zögerlich teil, wahrscheinlich aus krankheitsbezogener Zukunftsangst. Doch nach der Schulung kommt oftmals die Anfrage vonseiten der Teilnehmer „Wieso wird diese Schulung nicht mehr bekannt gemacht? Es ist doch eine hervorragende Sache und hilft mir sehr. Vielen Dank“.

Das Fundament für eine gute Patienteninformation ist durch den Kurs „Fit für Dialyse“ gelegt, aber es bedarf einer besseren Zusammenarbeit zwischen den Dialyse- und Nierentransplantationszentren, den Veranstaltern sowie den Referenten, um den Bekanntheitsgrad zu steigern. Die investierte Zeit und Mühe sollte noch effektiver genutzt und die Absprachen untereinander optimiert werden.

Fragen wie „Warum ich? Wie soll es weiter gehen? Welche Therapie passt zu mir?" geklärt werden. Erfahrene Patienten vermitteln Tipps und Tricks für den Alltag, Probleme werden besprochen und Sozialarbeiter erörtern Hinweise für sozialrechtliche Angelegenheiten.

Das neu gewonnene „Know how“ fördert die Compliance der Patienten und die Erkenntnis „Ich bin nicht allein“ kann reifen. Es ist unser Bestreben, den Patienten die Möglichkeit anzubieten, sich optimal auf die Zukunft vorzubereiten. Die Schulung ist als Wegweiser zu verstehen: Sie zeigt Optionen auf, aber der Patient muss sich selbst entscheiden, welchen Weg er gehen will - diese Entscheidung kann ihm nicht abgenommen werden.

\section{Ziel: bundesweite Anerkennung als Pflichtveranstaltung}

Das Hauptbestreben der Veranstalter ist es, die Patientenschulung „Fit für Dialyse“ deutschlandweit als Pflichtveranstaltung durchzusetzen, vergleichbar mit den schon seit Jahren etablierten Diabetikerschulungen. Eine komplette Kostenübernahme durch die Krankenkassen ist dabei unabdingbar. Denn nur ein informierter Patient kann gut mit der Erkrankung und den sich daraus ergebenden Problemen umgehen: $\mathrm{Er}$ ist offener für eventuelle Möglichkeiten und Konzepte.

\section{Den Patienten zu mehr}

\section{Eigenbeitrag motivieren}

Ziel der Wissensvermittlung ist es, den Patienten in die Lage zu versetzen, einen Eigenbeitrag zu leisten. Auf einfachste Art und Weise lernt der Patient, seine Gesundheit im Alltag zu stabilisieren und zu verbessern. Dadurch kann er beispielsweise Medikamentennebenwirkungen, die häufig schon in der Frühphase auftreten, schneller entgeper besser kennengelernt hat. Davon profitieren alle: der Patient mit seinen Angehörigen, das Pflegepersonal und das Ärzteteam - das Eis wird gebrochen. Wir wünschen uns, dass das jeweilige Dialysezentrum jedem präterminalen, niereninsuffizienten Patienten rät, an der Schulung „Fit für Dialyse“ teilzunehmen. Die Patienten haben die Schulung dankbar angenommen, diskutierten sehr angeregt und nahmen sie zum Anlass, ihren Kontakt zum Dialysezentrum zu intensivieren. Wir als Pflegepersonal sind mit verantwortlich, wenn es um die Patientencompliance geht. Ein informierter Patient ist offener für Gespräche, Körperkontrolle und Problembewältigung.

Elke Pforte, Jena genwirken, weil er sich und seinen Kör- 